Revue de l'Institut des langues et cultures

d'Europe, Amérique, Afrique, Asie et Australie

43 | 2021

Images des Amériques : fabrique, représentations, usages

\title{
Exotisme, civilisation et propagande : images de la ville de Rio de Janeiro au XIX ${ }^{\mathrm{e}}$ siècle
}

Exoticism, Civilization and Propaganda: Images of the City of Rio de Janeiro in the 19th Century

\section{Ana Maria Tavares Cavalcanti}

\section{OpenEdition}

\section{Journals}

Édition électronique

URL : https://journals.openedition.org/ilcea/13260

DOI : $10.4000 /$ ilcea. 13260

ISSN : 2101-0609

Éditeur

UGA Éditions/Université Grenoble Alpes

Édition imprimée

ISBN : 978-2-37747-299-4

ISSN : 1639-6073

\section{Référence électronique}

Ana Maria Tavares Cavalcanti, «Exotisme, civilisation et propagande : images de la ville de Rio de Janeiro au xix ${ }^{\mathrm{e}}$ siècle », ILCEA [En ligne], 43 | 2021, mis en ligne le 30 juin 2021, consulté le 30 juin 2021. URL : http://journals.openedition.org/ilcea/13260 ; DOI : https://doi.org/10.4000/ilcea.13260

Ce document a été généré automatiquement le 30 juin 2021.

(C) ILCEA 


\title{
Exotisme, civilisation et propagande : images de la ville de Rio de Janeiro au XIX ${ }^{\mathrm{e}}$ siècle
}

\author{
Exoticism, Civilization and Propaganda: Images of the City of Rio de Janeiro in \\ the 19th Century
}

Ana Maria Tavares Cavalcanti

1 Quelle image les Brésiliens et les Européens se faisaient-ils de la ville de Rio de Janeiro au début du XIX $\mathrm{X}^{\mathrm{e}}$ siècle ? Et comment cette image s'est-elle modifiée tout au long de ce siècle jusqu'au début du $\mathrm{xx}^{\mathrm{e}}$ ? Ces deux questions guident notre réflexion sur les représentations de la ville qui a été la capitale du Brésil de 1763 à $1910^{1}$.

Mais pourquoi démarrer notre recherche au XIx siècle? N'y a-t-il pas eu de représentations de la ville avant cela? En fait, en 1763, Rio de Janeiro est devenue la capitale du Brésil, alors colonie portugaise. Cependant, les artistes de cette période au Brésil produisaient soit des portraits, soit de l'art religieux destiné à orner les églises. Les images qui montraient la vie quotidienne dans la ville étaient très rares. D'autre part, à ce moment-là, les étrangers n'étaient pas autorisés à visiter le pays.

En 1808 cette situation a changé. C'est l'année où la famille royale portugaise est arrivée au Brésil avec la cour, fuyant les troupes napoléoniennes qui envahissaient le Portugal. La permission d'entrée a alors été accordée aux étrangers avec le Décret d'ouverture des ports brésiliens aux nations amies, promulgué par le prince régent Dom João VI après son arrivée au Brésil. Profitant de cette opportunité, les étrangers sont venus nombreux. Motivés soit par des buts commerciaux, soit par intérêt scientifique ou artistique, beaucoup d'entre eux ont laissé des récits ou des images où ils ont commenté leurs expériences à Rio de Janeiro. De son côté, l'élite brésilienne n'a pas toujours agréé ces témoignages. Lorsqu'il s'agissait de dévoiler leur capitale aux yeux du monde, les Brésiliens des classes favorisées s'inquiétaient de l'aspect plus ou moins civilisé des images présentées. 
Pour penser ces différents regards des Brésiliens et des étrangers sur Rio, sans nous perdre dans une multiplicité de représentations, nous avons mené notre réflexion à partir de trois repères, à savoir :

- le Panorama de Rio de Janeiro exécuté par Frédéric Guillaume Ronmy d'après les dessins et aquarelles préliminaires réalisées par Félix-Émile Taunay, exposé à Paris en 1824 ;

- les lithographies faites d'après les aquarelles de Jean-Baptiste Debret sur la ville de Rio de Janeiro, publiées dans le deuxième tome du livre Voyage pittoresque et historique au Brésil, à Paris en 1835 ;

- le projet du Théâtre lyrique de Rio de Janeiro présenté par Gottfried Semper à l'occasion du concours ouvert en 1857.

Ce qui nous intéresse dans ces œuvres si diverses - toile peinte du panorama, lithographies publiées dans un ouvrage illustré, projet architectural-, ce sont les réactions qu'elles ont provoquées chez les Brésiliens et les Européens. Nous verrons que le panorama de 1824 et l'ouvrage de Debret notamment sont devenus des références très importantes pour l'image de Rio de Janeiro et attirent toujours l'attention des historiens. Quant à la troisième œuvre choisie - le projet d'un théâtre qui n'a pas été construit -, elle nous intéresse justement parce qu'elle n'a pas été retenue par le jury qui l'a jugée en 1857. C'est ce refus qui est significatif parce qu'il rend évident le décalage entre la vision de l'architecte européen et celle des Brésiliens qui composaient le jury.

\section{Le panorama de Rio de Janeiro exposé à Paris en 1824}

6 La toile du panorama de Rio n'existe plus. La grande peinture circulaire n'était conçue que pour la durée de l'exposition. Enlevée de son dispositif architectural, il était presque impossible de la conserver après la fin de l'événement. Mais nous pouvons accéder à la mémoire de ce qui fut ce panorama à partir des études en aquarelle, d'une gravure qui reproduit le panorama et des textes écrits sur l'exposition.

Figure 1. - Friedrich Salathé (1793-1860) : Panorama de Rio de Janeiro d'après le panorama peint à Paris par G. F. Ronmy à partir des dessins de Félix-Émile Taunay.

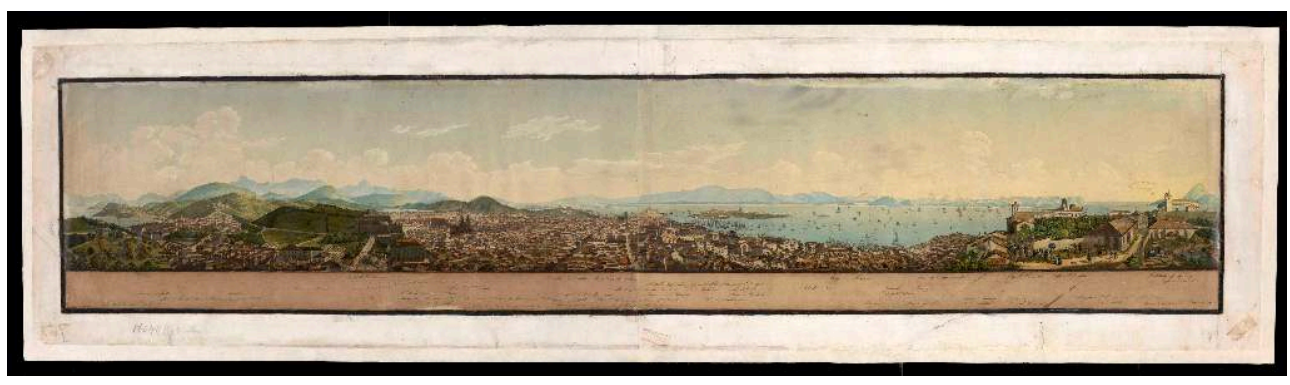

Aquatinte, $20 \times 100,5 \mathrm{~cm}$

Disponible sur : <http://objdigital.bn.br/acervo_digital/div_iconografia/icon408452/icon408452.jpg>.

7 En lisant les journaux de l'époque ${ }^{2}$, on apprend qu'au mois de mai 1824, le Panorama de Rio de Janeiro a été ouvert à la visite des Parisiens tous les jours de dix heures du matin à cinq heures du soir, à la rotonde du boulevard des Capucines. Cette rotonde, construite à l'initiative de Pierre Prévost (1764-1823), mesurait 32 mètres de diamètre 
et 16 mètres de hauteur. Ses grandes dimensions contribuaient à procurer l'illusion si prisée par le public. Pendant presque cinq mois le panorama de Rio est resté ouvert et a été très apprécié des visiteurs ${ }^{3}$.

On venait voir le spectacle tant pour s'amuser que pour s'instruire. Une Notice historique et explicative du panorama de Rio Janeiro a été publiée à cette occasion pour renseigner le public. Cette notice avait été écrite par deux Français qui connaissaient bien la ville et le pays : Hippolyte Taunay (1793-1864) et Ferdinand Denis (1798-1890). Hippolyte était le frère de Félix-Émile Taunay (1795-1881), qui avait fait les dessins et aquarelles préparatoires du panorama. Les deux hommes étaient venus au Brésil en 1816 pour accompagner leur père, le peintre Nicolas-Antoine Taunay, qui faisait partie du groupe d'artistes français engagés par D. João VI pour créer l'Académie des Beaux-Arts à Rio de Janeiro. Hippolyte est resté trois ans au Brésil. Outre Rio, il a connu les provinces de Pernambuco et Bahia. C'est à Salvador de Bahia qu'il a rencontré Ferdinand Denis, jeune voyageur devenu, par la suite, un historien du Brésil. Les deux amis, en rentrant en France, ont publié ensemble le livre Le Brésil ou Histoire des Mours, Usages et Coutumes des Habitants de ce Royaume (1822).

Dans la notice du panorama, ils font l'apologie du pays et commencent leur texte en présentant le Brésil et ses caractéristiques géographiques. Le panorama de la capitale brésilienne était en quelque sorte une carte de visite pour le pays tout entier. Parfois, le ton est celui d'une publicité touristique ou commerciale :

Quel pays offre les mêmes avantages? Jamais d'épidémie, point de volcans, et une fertilité qui fait rendre à la terre cent cinquante, et en quelques lieux, jusqu'à deux cent pour un.

Le port de Rio-Janeiro est si favorablement placé sur le globe, que les navires allant d'Europe dans la Mer du Sud ou dans celle des Indes, le rencontrent sur la route, vers la moitié du cours de leur navigation, et peuvent y renouveler leurs provisions épuisées ou avariées; aussi beaucoup de voyageurs l'ont-ils visité [...]. Tous s'accordent à louer en termes pompeux cette vaste baie, entourée de montagnes pittoresques, et par leurs formes variées, et par la verdure brillante qui les couvre et qui tranche avec le brun de la roche granitique dont elles sont formées. (Taunay \& Denis, $1824: 43-44)$

10 C'était la première fois que les Européens voyaient un paysage brésilien reproduit dans un panorama. On remarque que Ferdinand Denis et Hippolyte Taunay ont voulu présenter ce nouveau monde de façon très favorable au Brésil. Dans cet esprit, ils affirmaient encore :

[...] après avoir fourni sur les mœurs et sur les usages des habitans [sic] de cette capitale toutes les lumières qu'une longue habitude du pays nous a communiquées, nous terminerons par quelques détails principaux sur l'histoire naturelle d'un pays qui, par sa position avantageuse sur le globe, par l'étonnante fertilité de son territoire et par la splendeur à laquelle il est déjà parvenu, mérite assurément d'exciter la plus vive curiosité. (Ibid. : VI)

On perçoit dans cette notice explicative, une invitation adressée aux Européens à venir visiter, voire s'installer au Brésil. Les deux auteurs agissent, en quelque sorte, comme des représentants du nouveau gouvernement brésilien. L'indépendance du Brésil était très récente et pas encore reconnue par toutes les nations. En accord avec cette idée, il est intéressant de signaler que les artistes ont peint, dans le panorama, les figures de Dom Pedro I, de l'impératrice Léopoldine et de José Bonifácio de Andrada e Silva, les trois personnages clés de l'indépendance du Brésil ${ }^{4}$. Cette inclusion nous montre que le 
panorama de Rio, outre le but commercial, avait un but politique : présenter le pays dans son nouveau statut de nation indépendante.

Penchons-nous maintenant sur la réception du panorama par le public français, en commençant par les mots d'un contemporain qui s'est exprimé sur cet art et, en particulier, sur le panorama de Rio :

Une des inventions modernes les plus ingénieuses est sans contredit celle du panorama, qui, dans un tableau circulaire, offre au spectateur la vue la plus complète et la plus vraie des villes et des sites remarquables situés dans toutes les parties du globe. C'est ainsi que le public parisien a fait connaissance successivement avec Rome, Florence, Naples, Athènes, Jérusalem, etc. Aujourd'hui, c'est une nature nouvelle que l'on montre à ses regards, et il faut avouer que, sous ce rapport, le panorama de Rio-Janeiro mérite d'exciter la curiosité. [...] Rien de plus magnifique que la baie de Rio-Janeiro. Entourée de rochers gigantesques, qui abritent les vaisseaux contre les vents, elle est semée d'îles charmantes servant de promenade aux habitans. [...] La foule qui se porte chaque jour au boulevard des Capucines prouve que le public apprécie le talent de MM. Ronmy et Taunay fils, qui, appelés à faire valoir l'héritage de M. Provost [sic], promettent déjà de le remplacer dignement. (Lettres champenoises, $1824: 44-45$ )

D'après cet auteur, ce qui fascinait le public était la nature tropicale de Rio, différente de tout ce qui était connu en Europe. Les Parisiens avaient déjà vu les panoramas de Rome, Florence, Naples, Athènes, Jérusalem, et avaient été charmés par leurs monuments d'art, leurs constructions et œuvres architecturales qui avaient traversé des siècles d'histoire. Rio de Janeiro excitait la foule pour un tout autre motif. C'était surtout sa baie magnifique qui attirait les curieux. C'est justement ce qu'un journaliste a exprimé au sujet du panorama en question, à la section des Beaux-Arts du Journal des débats politiques et littéraires, dans un article intitulé « Panorama de Rio-Janeiro »:

Rio-Janeiro, située sous le beau ciel des tropiques et baignée par des mers qui ne repoussent pas, comme celles de la plupart de nos côtes, les richesses de la végétation, étoit un bon sujet de panorama, moins par elle-même que par le paysage pittoresque qui l'environne.

Ces villes du nouveau continent n'ont pas encore, et probablement n'auront jamais, pour l'observateur et pour l'ami des arts, le même degré, ou du moins le même genre d'intérêt que celles de l'ancien. [...] Pour eux, point de temps héroïques, point de vieil âge, point de siècles florissans des arts. [...]

Rio-Janeiro [...] est encore aujourd'hui plus semblable au champ d'une foire couvert de barraques [sic] qu'à la capitale d'un grand empire : c'est un amas confus de petites maisons, semblables de formes et de couleur, mal alignées le long des rues étroites et tortueuses. [...] point de monumens, si ce n'est quelques églises, toutes à peu près dans le même goût [...]. [...], sous le nom d'hôtels et de palais, les demeures $\mathrm{du}$ souverain et de ses ministres se distinguent des édifices particuliers par l'étendue du terrain qu'ils occupent, plutôt que par leur architecture. (Journal des débats politiques et littéraires, 25 juin 1824, p. 4)

Visiblement, l'auteur de ces lignes n'a pas été favorablement impressionné par les maisons, palais, églises et rues de Rio de Janeiro, surtout lorsqu'il les compare aux constructions des villes européennes. Pour lui, Rio était une ville sans histoire à laquelle manquait une architecture distinguée. Mais, dans la suite de son texte, il souligne les aspects du paysage :

Cette ville, ainsi qu'on vient de le voir, n'a rien de plus curieux, ni même de si curieux que la plupart de celles de l'Europe; mais le paysage est superbe en lui-même et tout nouveau pour nous : une mer brillante de lumière, parsemée d'îles charmantes, et doucement animée par les scènes variées de la navigation; les monticules du rivage, et ceux qui s'élèvent en amphithéâtre autour de la ville, 
couverts de palmiers, de bananiers et de toute cette belle végétation des tropiques, à travers laquelle s'aperçoit de loin en loin la muraille blanche de l'habitation du planteur; une atmosphère, des accidens de nuages et de lumière qui ne sont pas ceux de notre ciel, mais qu'on dit fidèlement imité de celui des zones torrides; tout cela, développé sur une toile de trois cent trente pieds, qu'agrandit encore à volonté l'illusion propre au Panorama, forme un fort beau tableau paysage et un spectacle fort curieux. (Ibid.) lithographies qui illustrent le deuxième tome du Voyage pittoresque présentent des similitudes et des différences qu'il importe de souligner. Aussi bien le panorama que les vues de Rio de Debret sont en rapport avec la venue d'artistes français au Brésil en 1816. On a déjà signalé que Félix-Émile Taunay et Hippolyte Taunay étaient fils de Nicolas-Antoine Taunay, peintre paysagiste membre du groupe engagé pour organiser l'enseignement artistique dans une Académie des Beaux-Arts crée à Rio de Janeiro. Jean-Baptiste Debret faisait partie du même groupe, plus tard identifié comme la «mission artistique française ». Un autre point commun est le public auquel le panorama et le Voyage pittoresque s'adressaient. Dans les deux cas, il s'agissait d'un public européen, plutôt français. artistes sur la ville. La vision panoramique à vol d'oiseau de 1824 contraste avec les scènes urbaines de Debret où l'on voit des personnages populaires dans les rues de Rio. La seconde différence à remarquer est celle du caractère permanent ou éphémère des images. Tandis que le panorama de Rio de 1824 n'existe plus physiquement, il en est autrement des images peintes par Debret. En fait, ses aquarelles et les lithographies réalisées d'après elles sont devenues des objets de collection, bien conservées dans des musées, exposées régulièrement, reproduites dans de beaux livres d'art au Brésil. 

de Janeiro, a été un grand succès de critique et de public. Les nombreux visiteurs se pressaient pour admirer les 120 aquarelles, une sélection parmi plus de 500 œuvres de Debret appartenant à la collection Castro Maia.

21 décennies que ces images sont largement diffusées dans des livres didactiques, des journaux et magazines, étant devenues des références utiles aux cinéastes et aux producteurs de feuilletons télévisés dont l'action se déroule à l'époque du Brésil colonial. La diffusion de ces images auprès du grand public avait été souhaitée par Debret lui-même qui les avait faites reproduire pour illustrer son livre dont le troisième et dernier tome est paru en 1839 en France.

Cependant, un fait attire notre attention : la première édition en portugais de l'ouvrage n'a été publiée qu'en 1939, avec un délai d'un siècle par rapport à l'édition française.

3 Nous ne sommes pas les premiers à nous étonner de ce retard. En 1978, lors d'une nouvelle édition brésilienne du Voyage pittoresque organisée par l'Université de São Paulo (USP), le professeur Mário Guimarães Ferri écrivait dans la préface : «[...] sa parution entre nous, en langue portugaise, a eu lieu seulement un siècle après la publication du dernier volume en France ${ }^{5}$. » La raison de ce retard, selon Ferri, fut la réception très négative de l'ouvrage par l'élite brésilienne contemporaine du peintre : « Des membres de l'Institut historique et géographique brésilien ont jugé "bouleversant que des coutumes des esclaves et des scènes de la vie populaire soient peints avec un tel réalisme"6.» $1978: 13)$

Tout porte à croire que Debret, en peignant ces aquarelles au Brésil, pensait déjà à leur future publication en Europe. Cette production n'était pas la motivation principale de son séjour au Brésil. Comme membre de la "mission artistique française », outre son travail comme professeur de l'Académie impériale des Beaux-Arts, Debret a exécuté des portraits des souverains et a peint des événements historiques tels que l'arrivée de l'archiduchesse Léopoldine à Rio de Janeiro, ou le couronnement de Dom Pedro I. Celles-ci étaient ses œuvres "officielles », celles que les Portugais et les Brésiliens, ses contemporains, connaissaient. Apparemment, il n'a pas montré aux membres de la cour, ni aux artistes locaux, ses aquarelles peintes dans les rues de Rio. C'est Debret lui-même qui affirme, dans l'introduction de son livre, que l'idée de publier ces images lui est venue après les avoir montrées à des étrangers de passage à Rio de Janeiro. Ces visiteurs l'auraient encouragé à les diffuser en Europe (Debret, 1834 : II).

En fait, les Européens étaient curieux du Brésil, ce «nouveau » monde accessible aux étrangers depuis 1808. Cette curiosité était la raison des nombreuses expéditions scientifiques en quête de nouvelles connaissances. Parmi les chercheurs venus au Brésil dans les décennies de 1810 et 1820 , nous pouvons citer le botaniste français SaintHilaire (1772-1845), les naturalistes bavarois Spix (1781-1826) et Martius (1794-1868) et le Russe Langsdorff (1774-1852), lesquels ont peut-être rencontré Debret au Brésil.

Après la parution du premier volume en 1834, Debret a envoyé un exemplaire à Dom Pedro II (Leenhardt, 2013 : 515). À cette occasion, l'Institut historique et géographique brésilien (IHGB) a pris en charge l'analyse du livre. Une commission d'experts l'a examiné et le volume a été accepté dans la bibliothèque de l'Institut. Le professeur Jacques Leenhardt a écrit à ce propos :

[...] ce premier volume se réfère aux habitants autochtones du territoire brésilien, ceux qui étaient appelés en Europe des «indiens» ou «sauvages». [...] orné de 
lithographies d'une grande qualité [...] coloriées à la gouache, a été bien reçu par les experts de l'IHGB et a été facilement accepté dans les collections impériales? (2013: 515) Debret dévoilait un pays peu civilisé aux yeux des étrangers. Trois estampes ont été spécialement critiquées par les membres de l'Institut. La première est celle qui montre " un employé du gouvernement sortant de chez lui avec sa famille». Bento da Silva Lisboa et Attaide Moncorvo, chargés de donner leur avis, ont vu dans cette image une caricature trompeuse sur les coutumes brésiliennes. En même temps qu'ils admettaient qu'en 1816, l'année de l'arrivée de Debret à Rio, « les coutumes n'avaient pas encore le degré de civilisation d'aujourd'hui ${ }^{10}$ ", ils affirmaient qu'on n'avait jamais su que les « employés du gouvernement sortaient pour se promener, en amenant leurs épouses dans leurs derniers mois de grossesse, conformément à ce que l'on voit dans l'estampe $\mathrm{e}^{11} »$ (Lisboa \& Moncorvo, $1841:$ 98).

Figure 2. - Jean-Baptiste Debret : Un employé du gouvernement sortant de chez lui avec sa famille.

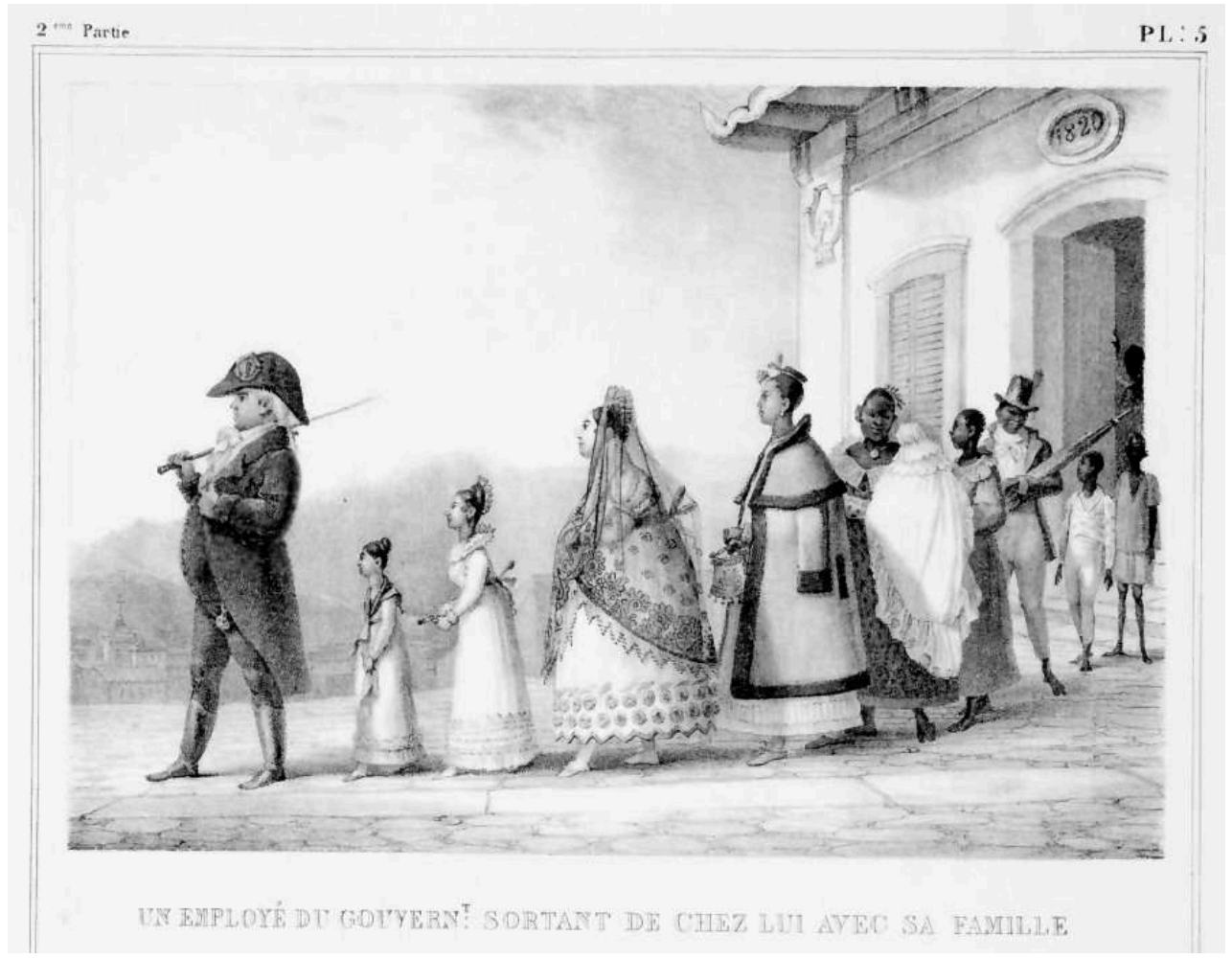

Planche n 5 du deuxième tome de Voyage pittoresque et historique au Brésil.

Les deux autres gravures sévèrement critiquées par les membres de l'IHGB ont été Boutique de la rue du Val-Longo et Feitors corrigeant des nègres.

La première a été considérée comme une caricature exagérée parce que Debret a mis en contraste un commerçant au gros ventre assis sur sa chaise au haut dossier, avec des 
esclaves cadavériques, nouvellement débarqués, assis par terre ou sur de longs bancs en bois.

Figure 3. - Jean-Baptiste Debret : Boutique de la rue du Val-Longo.

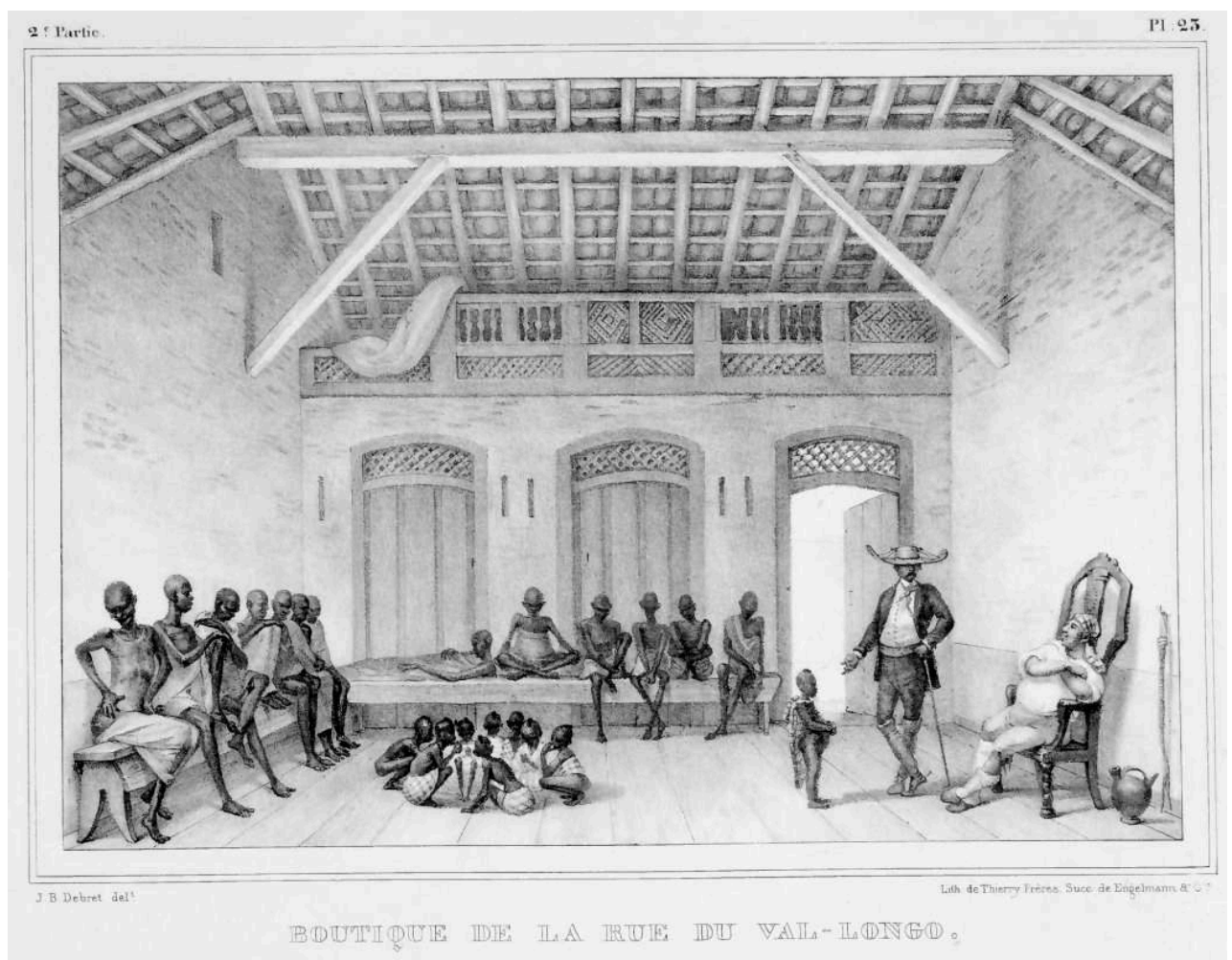

Planche nº 23 du deuxième tome de Voyage pittoresque et historique au Brésil.

Pour justifier leur critique, les membres de l'Institut ont fait la remarque suivante : « Le dessin que Mme Graham présente dans ses Voyages au Brésil est très différent ; puisqu'il est fait avec du sérieux et de la véracité ${ }^{12}$.» (Lisboa \& Moncorvo, 1841:98) Ainsi se référaient-ils au dessin de l'Anglais Augustus Earle qui montrait, lui aussi, le marché du Valongo. Ce dessin a été publié dans le livre de Maria Graham, Journal of a Voyage to Brazil, and Residence There, During Part of the Years 1821, 1822, 1823. 
Figure 4. - Augustus Earle : Valongo, ou marché d'esclaves à Rio.

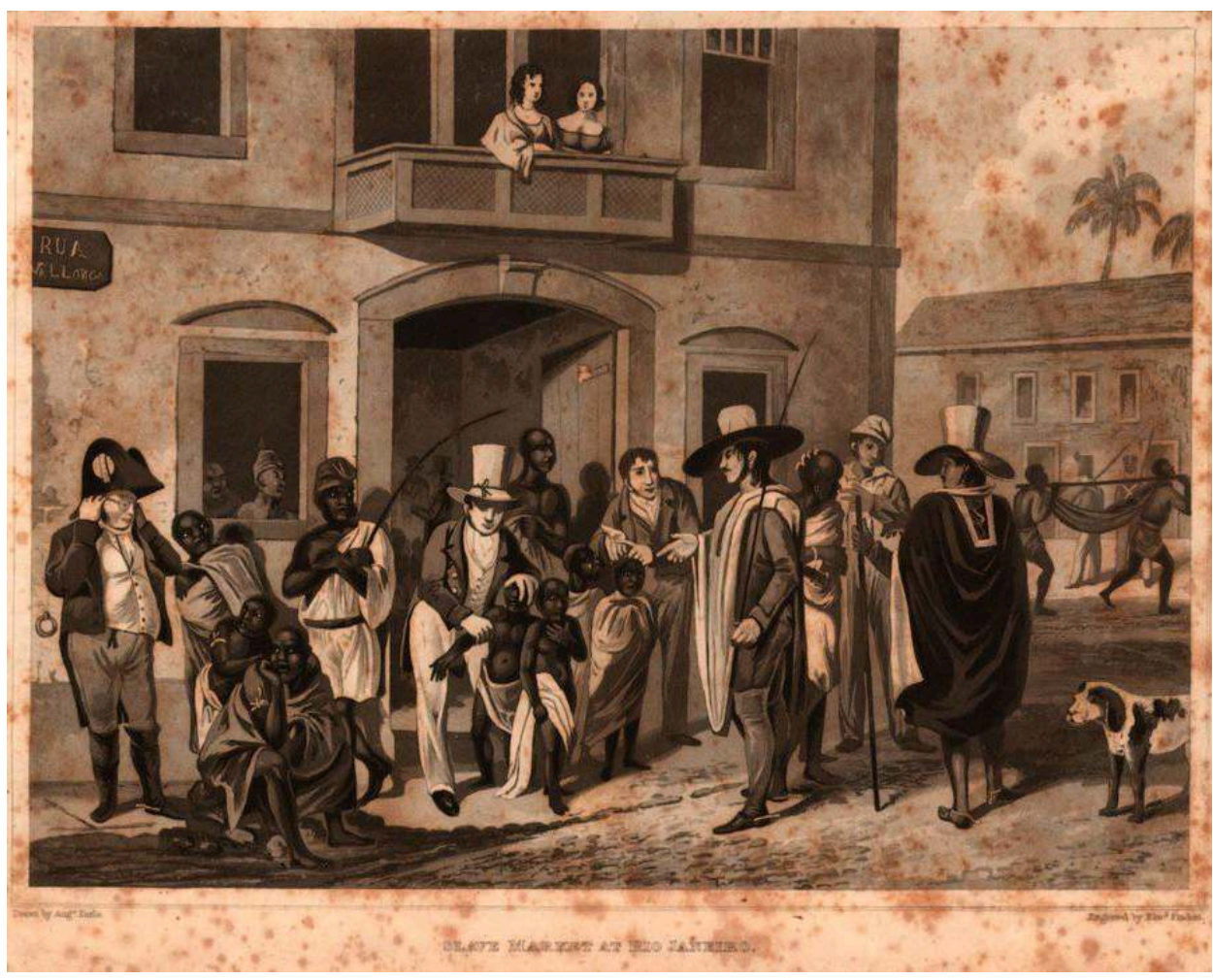

Source : M. Graham, Journal of a Voyage to Brazil, and Residence There, During Part of the Years 1827, 1822, 1823, Londres : Longman, 1824.

Il est compréhensible que les membres de l'IHGB aient préféré cette image du livre de Maria Graham à celle de Debret. Tandis que l'artiste français représente l'intérieur du marché sans aucune scène accessoire, le peintre anglais nous amuse avec une série de personnages secondaires. Ainsi, l'achat et la vente des esclaves sont dilués dans la vie mouvementée de la ville fixée par Earle. 
Figure 5. - Jean-Baptiste Debret : Feitors corrigeant des nègres.

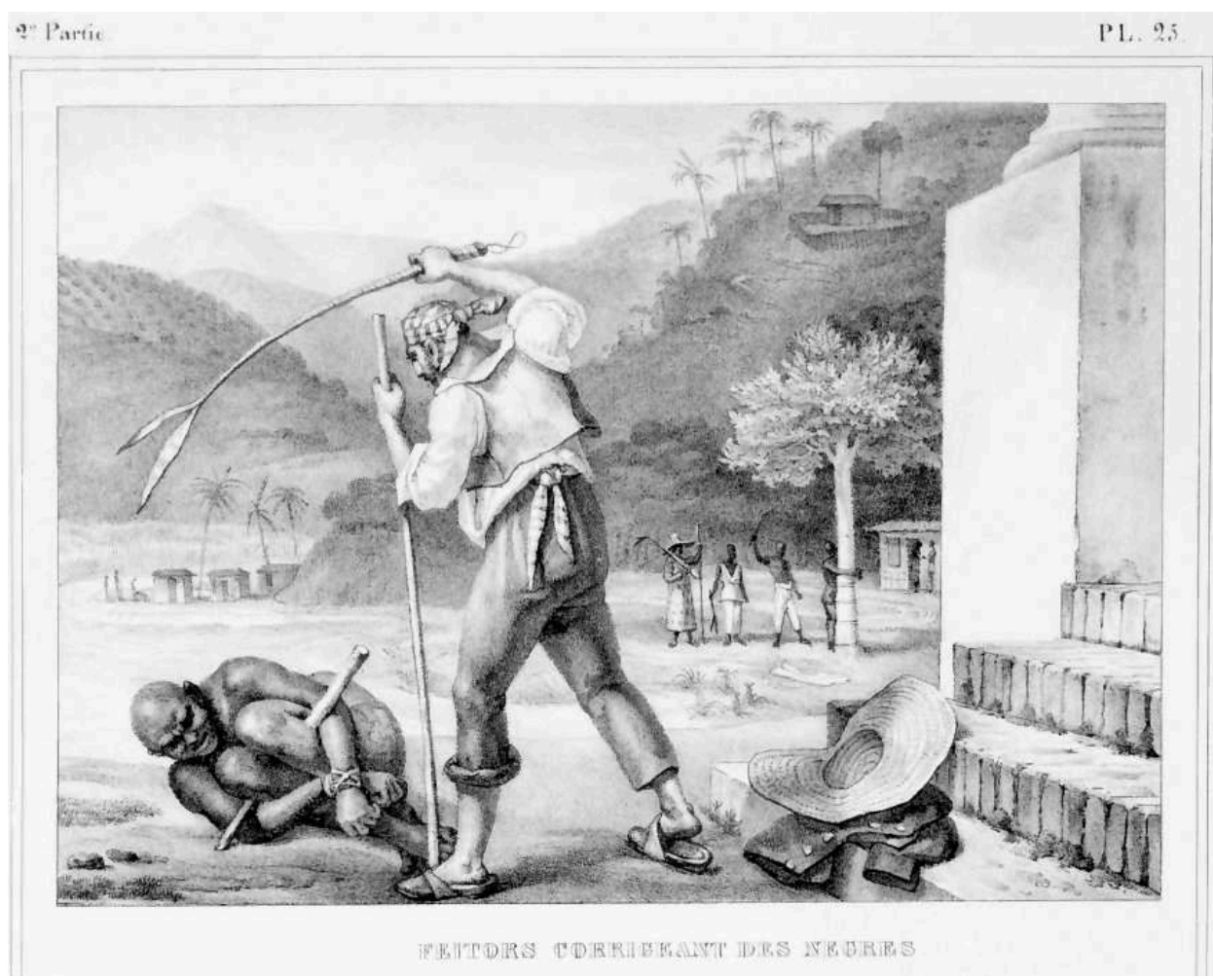

Planche nº 25 du deuxième tome de Voyage pittoresque et historique au Brésil.

Au sujet de la deuxième image, Feitors corrigeant des nègres, la commission a fait la remarque suivante: "L'attitude du patient [...] provoque l'horreur. Il se peut que M. Debret ait vu un châtiment semblable, parce que partout il y a des seigneurs barbares ; mais cela n'est qu'un abus ${ }^{13}$.» (Lisboa \& Moncorvo, 1841 : 98) L'argument ne nous semble pas convaincant.

Nous sommes en présence de l'embarras des intellectuels brésiliens membres de l'IHGB par rapport au deuxième tome de Voyage pittoresque dû au fait qu'il dévoilait aux yeux de tous une image du Brésil qu'ils ne voulaient pas voir, et surtout qu'ils ne voulaient pas faire connaître aux étrangers. Même dans un article favorable au livre, publié dans le Jornal dos debates politicos e litterarios, le 27 mai 1837, Gonçalves de Magalhães ${ }^{14}$, après avoir fait des éloges sur le premier tome, dans lequel il s'agit des peuples indigènes, se montre laconique à propos du deuxième, en disant: «Le $2^{\mathrm{e}}$ volume contient les us et coutumes du peuple, dont certains, avec le progrès de la civilisation, sont en train de disparaître ${ }^{15}$. » (Jornal dos debates, 27 mai 1837, p. 31).

Le progrès de la civilisation compris comme l'européanisation du pays, voilà l'aspiration d'une élite brésilienne contrariée par l'exhibition des horreurs de l'esclavage étalés par Debret dans ses aquarelles. Cette civilisation tant désirée n'impliquait pas forcément de vrais changements, mais devait se faire voir dans l'architecture de la ville, comme nous pourrons le constater dans le prochain cas analysé. L'urbanisme irrégulier de Rio de Janeiro avait déjà été remarqué par les Français qui avaient critiqué l'amas confus des maisons et rues tortueuses de la ville, dans le panorama présenté à Paris en 1824. Dans la suite des images choisies pour nous servir de repère, nous allons examiner les projets pour un nouveau théâtre qui devait être construit au cœur de la ville de Rio de Janeiro. C'était en 1857, et l'architecture 
représentait un espoir, pour l'élite brésilienne, pour changer l'aspect colonial de la capitale de l'Empire.

\section{Le Théâtre lyrique de Rio de Janeiro par Gottfried Semper}

C'était la première fois qu'un concours d'architecture était organisé par le gouvernement impérial brésilien. Le besoin d'un nouveau théâtre lyrique était la conséquence de l'incendie qui avait détruit le Real Teatro de São João, situé sur la Praça da Constituição (actuelle Praça Tiradentes), en 1851. Cette même année, Guilherme Schüch et Manuel de Araújo Porto-Alegre avaient demandé à l'empereur Dom Pedro II d'ouvrir un concours d'architecture pour un nouveau théatre ${ }^{16}$. Six ans plus tard, ce souhait allait se réaliser. Le programme du concours, publié le 13 novembre 1857 à la une du Jornal do Commercio, informait des exigences à propos de la construction ${ }^{17}$. La façade principale de l'immeuble donnerait sur la Praça da Aclamação, aujourd'hui Praça da República. Le théâtre ne se destinerait pas seulement à l'exhibition de grands spectacles, tels que les opéras lyriques et les ballets, mais aussi à la réalisation des fêtes publiques. Une loge impériale au fond de la salle, pour les jours de gala, était expressément demandée ; et pour les autres jours, la famille impériale aurait une loge à côté du proscenium. Le théâtre devrait être « construit selon le système moderne, et adapté au climat de Rio de Janeiro ${ }^{18}$ », et surtout, le jury n'admettrait pas « des copies ou imitations serviles d'autres théâtres ${ }^{19} »$. Dans le programme du concours, la participation des architectes résidents à l'étranger était prévue. Ces concurrents auraient droit à deux mois supplémentaires pour présenter leurs projets. Pour tous les autres, le projet devrait être présenté dans les neuf mois et quinze jours après la publication du programme. Les candidats devraient signer leurs projets par un anagramme ou épigraphe, leurs vrais noms étant cachés dans des enveloppes qui seraient ouvertes après le jugement.

Le 23 mars 1859, le résultat du concours a été publié dans le même Jornal do Commercio ${ }^{20}$. Par cette publication, nous savons que le jury a reçu vingt-huit projets. Dix-huit ont été disqualifiés parce qu'ils ignoraient le premier article du programme du concours qui fixait les limites du terrain où serait construit le théâtre. Le projet de Gottfried Semper (1803-1879), signé Ver non semper floret, se trouvait parmi ceux-là. Il nous importe tout de même de l'analyser. Gottfried Semper était un architecte reconnu en Europe. Il avait dessiné l'Opéra de Dresde (1837-1841) et était professeur d'architecture à l'École polytechnique de Zurich. Son projet de théâtre pour Rio est très significatif et différent du projet vainqueur, comme nous le verrons par la suite.

Des dix projets qui ont effectivement participé au concours, le jury a préféré à tous les autres celui signé Suum cuique, dont l'auteur était Gustav Waehneldt (1830-1873), architecte allemand établi à Rio de Janeiro depuis 1852 . Voyons donc les deux projets : 
Figure 6. - Gottfried Semper (1803-1879) : Projet pour un nouveau théâtre à Rio de Janeiro, 1858.

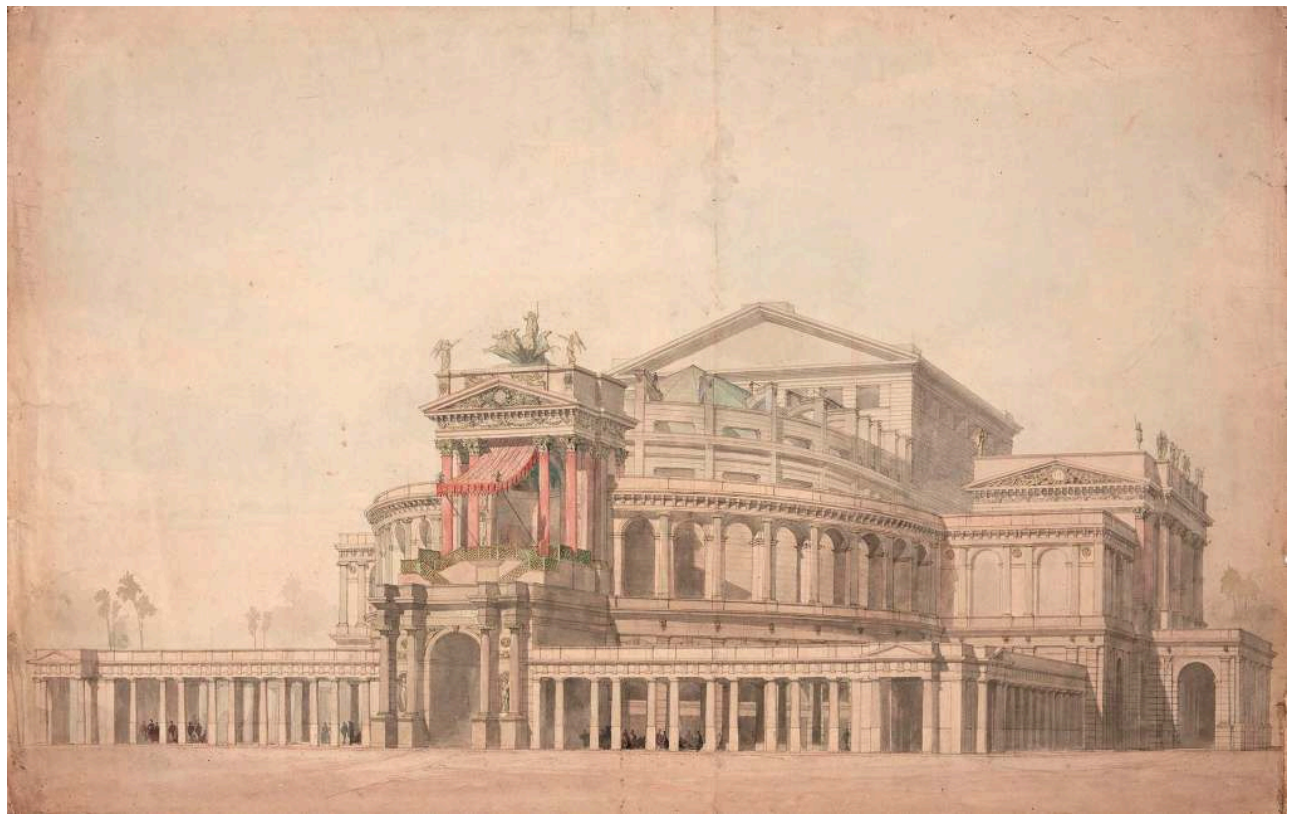

Munich, Deutsches Theatermuseum. Photo : Archiv/ETH Zurich.

Source : Gnehm (2017). Disponible sur : <www.ciha.org/sites/default/files/files/

NewWorldsFrontiersInclusionUtopias_2017.pdf>.

Figure 7. - Gustav Waehneldt (1830-1873) : Projet pour un nouveau théâtre à Rio de Janeiro.

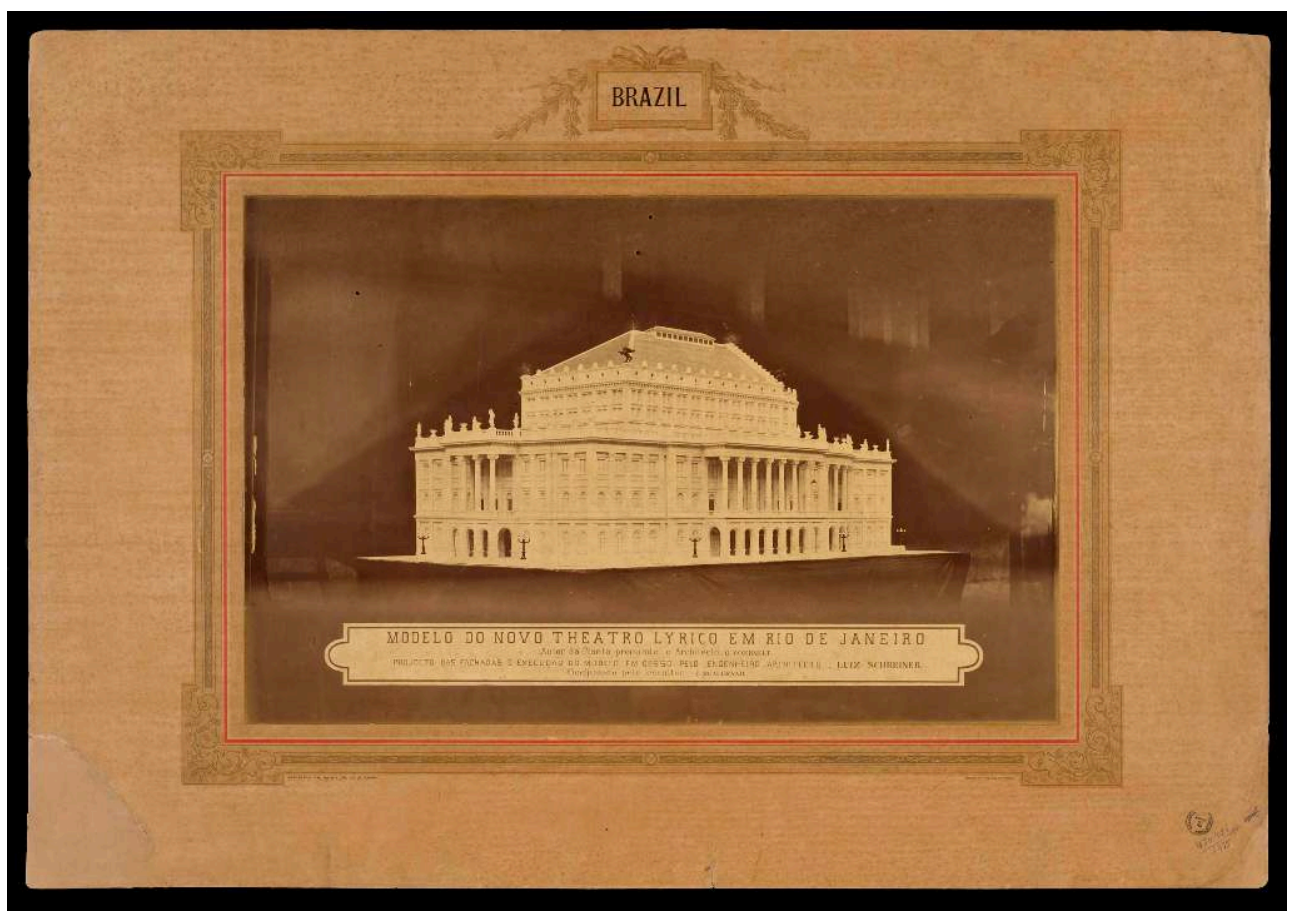

Modèle en plâtre par Luiz Schreiner, c. 1876, Rio de Janeiro.

Disponible sur : <http://objdigital.bn.br/acervo_digital/div_iconografia/icon470421/icon470421.jpg>.

39 Tout d'abord, observons le dessin très original de Semper. Il est vrai que ses volumes cylindriques et son portail central nous font penser à son Opéra à Dresde (1837-1841) avec son entrée monumentale. Mais le projet pour Rio de Janeiro est encore plus 
imaginatif, avec sa succession de façades qui se déploient en alternant des formes rectilignes et courbes. Dans les Notes explicatives du projet, rédigées en français, Semper a expliqué la raison du "double portique extérieur » formé par des «rideaux» de colonnades : "Sous un climat comme celui du Brésil [ce double portique] sera presque nécessaire, tout comme promenoir pendant les entractes des représentations et scéniques [sic]; que pendant la journée pour le public et surtout pour ceux qui attendront l'ouverture des caisses ${ }^{21}$." On voit qu'il a voulu, avec ses rideaux de colonnes, répondre à une des exigences du concours, c'est-à-dire adapter son théâtre au climat de Rio de Janeiro. Dans la suite de ses explications, Semper avance des raisons esthétiques pour ce double portique :

Mais ce qu'il s'agit surtout de faire ressortir par rapport à ces portiques c'est qu'ils seront non seulement utiles, mais qu'ils serviront d'échelle et de cadre pour mieux faire valoir les proportions de l'édifice qu'ils entourent. Celui-ci loin d'être caché derrière le rideau transparent de cette colonnade aréostyle, paraîtra plus distinctement par l'effet du contraste. Car il dépassera considérablement son entourage transparent [...] et se montrera plus grandiose ${ }^{22}$.

Cet aspect monumental et luxueux consciemment cherché par Semper est aussi présent dans un élément de la façade principale qui attire spécialement notre attention: la loggia impériale qui nous fait penser aux empereurs de la Rome antique.

Finalement, on remarque l'inédite présence des arcs-boutants qui supportent la structure du toit de l'auditorium, signalant une liberté formelle sans précédent et apportant des éléments d'architecture médiévale qui se mélangent au répertoire classique. Si l'on compare ce dessin au projet vainqueur présenté par Gustav Waehneldt, le théâtre de Semper apparaît comme une image de rêve qui sort des sentiers battus.

Penchons-nous maintenant sur le projet de Waehneldt. Il nous semble très classique avec ses suites de colonnes droitement alignées. Les formes sont simples et se répètent. Justement, c'est cette sobriété du projet de Waehneldt qui a plu aux jurés qui ont écrit à ce propos :

Plan $n^{\circ} 12$, noté Suum cuique. Ce plan satisfait à toutes les exigences du programme ; il offre toutes les conditions souhaitables de sécurité et de commodité; il se fait remarquable par la simplicité des ornements, surtout ceux de l'extérieur.

[...]. Selon ce jugement, le plan de l'anagramme Suum cuique est supérieur à tous les autres qui sont entrés en concours, et doit être préféré pour être construit ${ }^{23}$. Jornal do Commercio, 23 mars 1859, p. 1)

3 Cette affirmation du jury selon laquelle «ce plan [...] se fait remarquable par la simplicité des ornements » a été réfutée par un critique anonyme contemporain qui a signé «Un Concurrent », dans le premier article d'une série publiée à Rio de Janeiro dans le Correio Mercantil. Cet adversaire de Waehneldt disait que les ornements simples qui avaient reçu des compliments étaient vraiment «trop simples, puisqu'ils n'existent pas ! L'extérieur n'est qu'une superposition de pilastres ${ }^{24}$ » (Correio Mercantil, 26-27 mars 1859, p. 1).

Dans la continuation de son appréciation sur le projet vainqueur, le jury faisait une petite remarque qu'il est intéressant de souligner :

La commission l'apprécierait d'avantage s'il n'avait pas une façade cylindrique; mais ce petit hic ne contrarie en rien les clauses du programme, ni ne modifie le jugement unanime des membres de la commission ${ }^{25}$. (Jornal do Commercio, 23 mars 1859, p. 1) 

membres du jury du concours. S'ils ont signalé la façade cylindrique de Waehneldt comme le « petit hic » de son projet, ils auraient critiqué davantage la façade courbe de Semper, ainsi que la quantité et la diversité des ornements qu'il a dessinés. En fait, on peut lire dans leur jugement publié dans le Jornal do Commercio, plusieurs remarques qui indiquent une aversion des extravagances. Pour se référer aux défauts qu'ils ont rencontrés dans les façades des projets perdants, ils ont employé les expressions suivantes : "trop chargées d'ornements et des statues », " de nombreux ornements inutiles et dispendieux ", " construction difficile et chère, excessivement chargée de saillies », « façade surchargée d'ornements inutiles et dispendieux » ou " architecture excessivement chargée d'ornements dispendieux $»^{27}$ (Jornal do Commercio, 23 mars 1859, p. 1).

47 Le théâtre dessiné par Gottfried Semper pour Rio n'a jamais été construit, de même pour le théâtre de Waehneldt : les deux sont restés à l'état de projets. Pour des raisons économiques, le gouvernement brésilien a abandonné l'idée de cette construction. Cependant, ces deux projets nous intéressent àcause des idées qu'ils expriment à propos de ce qui devait être un théâtre au Brésil en 1858. Si différents l'un de l'autre, ils sont les résultats des pensées et des points de vue divergents. D'une part, le projet de Gottfried Semper révèle ses idées à propos d'une terre étrangère exotique. Le Brésil impérial de son imagination méritait un théâtre lyrique extraordinaire et exubérant. D'autre part, le projet vainqueur nous montre que les membres du jury du concours, tout en affirmant qu'ils souhaitaient un théâtre original et non pas « des copies ou imitations serviles d'autres théâtres », restaient attachés aux modèles grecs auxquels l'élite brésilienne se fiait pour se rapprocher de la civilisation de l'Europe et de ses liens avec la tradition classique.

\section{Sur les images de la ville de Rio de Janeiro et les idées d'exotisme et civilisation}

Après avoir traité ces trois exemples de représentations de la ville de Rio de Janeiro, quelles idées pouvons nous retenir?

Quant au Panorama de 1824, d'une part il a répandu une image idyllique d'un pays tropical lointain qui faisait rêver avec ses « îles charmantes " parsemées dans la baie. D’autre part, il a donné lieu à une vision qui montait en épingle l'absence de passé historique de la capitale brésilienne, ainsi que le manque d'œuvres architecturales de valeur, lorsque l'on comparait Rio aux plus importantes villes européennes. 
Quelques années plus tard, les aquarelles de Debret exposaient les aspects exotiques de Rio de Janeiro. Désormais, la ville qui dans le Panorama était vue de loin, se rapprochait et devenait vivante avec des scènes animées par des personnages populaires presque toujours noirs, esclaves ou libres, dans les rues de Rio. Les œuvres architecturales dessinées par Debret se présentaient toujours dans leur simplicité et leur uniformité, critiquées en 1824, mais composaient un paysage original en rendant évidentes leur particularité et leur différence par rapport aux villes européennes. L'élite brésilienne contemporaine a critiqué les dessins de Debret justement parce qu'ils montraient une ville exotique qui devait attirer le regard du public européen des années 1830, auquel ils s'adressaient.

51 Le projet de Gottfried Semper pour le théâtre lyrique de Rio en 1858 nous intéresse aussi parce qu'il signale la permanence de cette idée d'exotisme projetée par les Européens sur le Brésil, une idée qui n'était pas intégrée par les Brésiliens. En outre, le concours pour le théâtre, qui finalement n'a pas été construit, annonçait l'inquiétude des intellectuels qui voulaient embellir la capitale du pays avec une architecture significative.

Ce désir de remodeler Rio de Janeiro d'après le modèle européen s'est finalement mis en place avec les travaux de réaménagement de la ville au début du $\mathrm{xx}^{\mathrm{e}}$ siècle.

Les images du panorama de 1824 et du théâtre de 1858 ont disparu en laissant des traces de ce qu'elles ont été et des idées dont elles sont emblématiques. Elles n'existent plus, alors que les aquarelles de Debret sont plus vivantes que jamais. Les Brésiliens d'aujourd'hui voient ces images comme des documents visuels gardiens de la mémoire nationale.

remarque le changement d'attitude de l'élite brésilienne devant les images de Debret. Après presque deux siècles, elle a un regard curieux comme celui des Européens des années 1830.

Dans les images de Rio de Janeiro que nous venons d'analyser, le rapport entre les artistes et le public a contribué à créer un imaginaire collectif multiple où se sont mélangés la sensation d'exotisme et l'aspiration à la civilisation. Il importe de souligner que tout au long du XIX $x^{e}$ siècle, la ville s'est maintenue comme capitale du Brésil, villephare regardée comme un microcosme du pays. Ainsi, le désir d'égaler l'Europe et la valorisation des aspects pittoresques ont joué des rôles importants dans la formation d'une identité nationale brésilienne.

\section{BIBLIOGRAPHIE}

BANDEIRA Julio \& LAGo Pedro Corrêa (2009), Debret e o Brasil: obra completa, 1816-1831, Rio de Janeiro : Capivara.

DEBRET Jean-Baptiste (1834), Voyage pittoresque et historique au Brésil, ou séjour d'un artiste français au Brésil depuis 1816 jusqu'en 1831 inclusivement (t. 1), Paris : Firmin Didot frères. 
DEBRET Jean-Baptiste (1978), Viagem pitoresca e histórica ao Brasil, São Paulo : Editora da Universidade de São Paulo.

DiAs Elaine (2009), Paisagem e Academia: Félix-Émile Taunay e o Brasil (1824-1851), Campinas : Editora da Unicamp.

FERRI M. G. (1978), « Apresentação », J.-B. Debret, Viagem pitoresca e histórica ao Brasil, São Paulo : Editora da Universidade de São Paulo, 13-14.

GNEHM Michael (2017), « Tropical Opulence: Rio de Janeiro's Theater Competition of 1857 », C. M. Avolese \& R. Conduru (dir.), New Worlds: Frontiers, Inclusion, Utopias, São Paulo : CBHA / CIHA. Disponible sur <www.ciha.org/sites/default/files/files/ NewWorldsFrontiersInclusionUtopias_2017.pdf>.

GRAHAM Maria (1824), Journal of a Voyage to Brazil, and Residence There, During Part of the Years 1821, 1822, 1823, Londres : Longman. Disponible sur <http://purl.pt/17069/1/index.html\#/1/html>.

HERmann Carla (2013), « Considerações sobre dois panoramas viajantes do Rio de Janeiro no século XIX », Revista do Arquivo Geral da Cidade do Rio de Janeiro, 7, 105-117.

LAGo Pedro Corrêa do (2008), Taunay e o Brasil: obra completa, 1816-1821, Rio de Janeiro : Capivara. LEENHARDT Jacques (2013), « Jean-Baptiste Debret: um olhar francês sobre os primórdios do Império brasileiro », Sociologia \& Antropologia, 3(6), 509-523.

«Panoramas de Salon », Le Figaro, journal littéraire, 4(357), 23 décembre 1829, p. 1.

Lettres champenoises, ou correspondance morale et littéraire, rédigé par plusieurs hommes de lettres adressée à Madame de ${ }^{* * *}$ à Arcis-sur-Aube, 17(145), Paris : Pillet ainé, imprim.-libraire, 1824.

LisBoA Bento da Silva \& Moncorvo J. D. de Attaide (1841), « Parecer sobre o $1^{\circ}$ e $2^{\circ}$ volume da obra intitulada Voyage pittoresque et historique au Brésil ou séjour d'un artiste français au Brésil, depuis 1816 jusqu'en 1831 inclusivement, par J.-B. Debret ", Revista Trimensal de Historia e Geographia ou Jornal do Instituto Histórico Geographico Brasileiro, Rio de Janeiro : Typographia de D. L. dos Santos, réimpr. en 1860, 95-99. Disponible sur <https://www.ihgb.org.br/publicacoes/revista-ihgb/itemlist[...]>.

PATto Maria Helena Souza (1999), « Estado, ciência e política na Primeira República: a desqualificação dos pobres », Estudos Avançados, 13(35), 167-198.

PEREIRA Margareth da Silva (1994), « Romantismo e objetividade: notas sobre um panorama do Rio de Janeiro », Anais do Museu Paulista, nouv. série, 2 (jan./déc. 1994), 169-195, $<$ www.revistas.usp.br/anaismp/article/view/5299/6829>.

PREFEITURA DA CidADE Do Rio De JANEIRo (2002), Memória da destruição. Rio, uma história que se perdeu (1889-1965), Rio de Janeiro.

TAUNAY Hippolyte \& DENIS Ferdinand (1824), Notice historique et explicative du panorama de Rio Janeiro, Paris : Nepveu.

SCHWARCZ Lilia Moritz \& DiAs Elaine (2008), Nicolas-Antoine Taunay no Brasil: uma leitura dos trópicos, Rio de Janeiro : Sextante.

SouZA Thiago Leitão de (2014), o panorama e a experiência imersiva: do espetáculo de entretenimento aos meios digitais (thèse), Universidade Federal do Rio de Janeiro, Rio de Janeiro. 


\section{NOTES}

1. Je tiens à remercier Valentine Mercier et Inès Cavalcanti pour leur aide précieuse dans la révision de cet article.

2. On trouve des notices sur le Panorama de Rio de Janeiro de 1824 dans La Quotidienne, Le Constitutionnel, Le Courrier français, Le Corsaire, le Journal des débats politiques et littéraires, le Journal du Commerce, Lettres champenoises et dans le Panorama des nouveautés parisiennes.

3. On mesure l'appréciation des visiteurs du Panorama de Rio de Janeiro non seulement par la presse qui rapportait la présence d'un public nombreux, mais aussi par les multiples reproductions gravées de ce panorama qui se trouvent dans des collections privées (Hermann, 2013 : 112). Aussi, ce panorama a été repris par Auguste-Nicolas Nepveu lors de son entreprise commerciale du panorama-salon en 1829 (Le Figaro, 1829 : p. 1).

4. Les trois personnages apparaissent dans les aquarelles préliminaires du panorama de Rio de Janeiro (collection privée, France) et aussi dans la gravure de Friedrich Salathé qui reproduit le panorama de 1824 (Biblioteca Nacional, Rio de Janeiro).

5. Dans l'original en portugais : «[...] sua aparição entre nós, em língua portuguesa, ocorreu somente um século após a publicação do último volume na França. »

6. Dans l'original en portugais : "Membros do Instituto Histórico e Geográfico Brasileiro julgaram "chocante que se pintassem costumes de escravos e cenas da vida popular com tanto realismo". »

7. Dans l'original en portugais : "[...] este primeiro volume se refere aos habitantes autóctones da terra brasileira, os que eram chamados na Europa de "índios" ou "selvagens". [...] adornado por litografias de grande qualidade [...] coloridas a guache, é bem recebido pelos peritos do IHGB e entra facilmente para as coleções imperiais. "

8. Les avis de Bento da Silva Lisboa et J. D. de Attaide Moncorvo sur les deux premiers tomes de Voyage pittoresque et historique au Brésil datent du 18 juillet et du 31 octobre 1840, respectivement. Les deux avis ont été publiés dans la Revista Trimensal de Historia e Geographia, tome 3, en 1841. Disponible sur <https://www.ihgb.org.br/publicacoes/revista-ihgb/itemlist/filter.html? searchword438-from=1839\&searchword438-to=1841\&moduleId=219\&Itemid=174>.

9. Dans l'original en portugais : "lugar que ocupavam os escravos na vida cotidiana e no trabalho na cidade ».

10. Dans l'original en portugais : "os costumes não tinham adquirido aquele grau de civilização que hoje tem ".

11. Dans l'original en portugais: «empregados públicos saíssem a passeio, levando suas esposas no último período de gravidez, segundo se vê na estampa».

12. Dans l'original en portugais : «Bem diferente é o desenho que apresenta a Senhora Graham nas suas Viagens ao Brasil; pois que é feito com seriedade e veracidade. "

13. Dans l'original en portugais : «A atitude do paciente é tal que causa horror. Pode ser que M. Debret presenciasse semelhante castigo, porque em todas as partes há senhores bárbaros; mas isto não é senão um abuso."

14. Gonçalves de Magalhães (1811-1882) était médecin, professeur, diplomate, politicien, poète et écrivain brésilien. Avec Araújo Porto-Alegre et Torres Homem, il a créé la revue Nitheroy, revista brasiliense (1836), importante publication du début du romantisme au Brésil.

15. Dans l'original en portugais : "Contém o $2^{\circ}$ vol. os costumes e usos do povo, alguns dos quaes com o progresso da civilisação vão desaparecendo. "

16. Pétition du 12 août 1851, Rio de Janeiro, Arquivo Nacional, IE 743. Voir Gnehm (2017 : 147).

17. Ministério do Império, « Programma de concurso para construcção de um theatro na cidade do Rio de Janeiro ", Jornal do Commercio, année 32, n 312 (13 novembre 1857), p. 1. Disponible sur $<$ http://bndigital.bn.gov.br/hemeroteca-digital/>.

18. Dans l'original en portugais : « $4^{\circ}$ Será construido segundo o systema moderno, e adaptado ao clima do Rio de Janeiro ». 
19. Dans l'original en portugais : « não sendo admitidas cópias ou imitações servis de outros theatros ». 20. Ministério do Império, « Termo de julgamento ", Jornal do Commercio, année 34, n 82 (23 mars 1859), p. 1. Disponible sur <http://bndigital.bn.gov.br/hemeroteca-digital/>.

21. Gottfried Semper, « Notes explicatives au projet de Théâtre pour la ville de Rio de Janeiro, avec l'Épigraphe: Ver non semper floret », MS (1858), 3 (gta Archiv/ETH Zurich, 20-153-DOK-10). Cité d'après Gnhem (2018: 159).

22. Gottfried Semper, « Notes explicatives au projet de Théâtre pour la ville de Rio de Janeiro, avec l'Épigraphe: Ver non semper floret », MS (1858), 3 (gta Archiv/ETH Zurich, 20-153-DOK-10). Cité d'après Gnhem (2018:160).

23. Dans l'original en portugais : «Plano n.12, notado Suum cuique. Este plano satisfaz a todas as exigências do programa; oferece todas as condições desejáveis de segurança e comodidade; faz-se notável pela singeleza dos ornamentos, maxime os exteriores. [...] Segundo este juízo, o plano do anagramma Suum cuique é muito superior a todos os outros que entraram em concurso, e deve ser o preferido para a construção. »

24. Dans l'original en portugais : «são muito singelos os ornamentos, singelos demais até, pois que não existem! Não passa o exterior de uma superposição de pilastras ». Um concorrente, "Concurso para o theatro lyrico", Correio Mercantil, année 16, $\mathrm{n}^{\circ} 85$ (26-27 mars 1859). Cité d'après Gnhem (2018 : 152).

25. Dans l'original en portugais : « A comissão o estimaria mais se não tivesse a fachada cilíndrica ; mas este pequeno senão em nada contraria as cláusulas do programa, nem modifica o juízo unânime dos membros da comissão. "

26. Gottfried Semper, « Notes explicatives au projet de Théâtre pour la ville de Rio de Janeiro, avec l'Épigraphe : Ver non semper floret ", MS (1858), 3 (gta Archiv/ETH Zurich, 20-153-DOK-10). Cité d'après Gnhem (2018: 155).

27. Dans l'original en portugais : « em demasia sobrecarregadas de ornatos e de estátuas ", " contém numerosos ornatos de inútil e dispendioso trabalho", "construção difícil e dispensiosa, excessivamente carregada de ressaltos", "fachada sobrecarregada de ornatos inúteis e dispendiosos ", " arquitetura excessivamente carregada de ornatos dispendiosos ».

\section{RÉSUMÉS}

Quel rôle ont joué les idées d'exotisme ou de civilisation dans la représentation de la ville de Rio de Janeiro tout au long du XIX ${ }^{\mathrm{e}}$ siècle jusqu'au début du Xx ${ }^{\mathrm{e}}$ ? Quelles différences peut-on observer entre les images que Brésiliens et Européens se faisaient de la ville? Pour répondre à ces questions, nous nous intéressons aux réactions que diverses œuvres représentant la ville de Rio ont suscitées de part et d'autre de l'Atlantique. Ces exemples nous montrent combien ces points de vue ont pu s'influencer réciproquement. On se rend compte que le désir d'égaler l'Europe et la valorisation des aspects pittoresques ont eu une importance majeure dans la formation d'une identité nationale brésilienne.

What role did ideas of exoticism or civilization play in the representation of Rio de Janeiro throughout the nineteenth century and into the early twentieth century? What differences can we see in the way Brazilians and Europeans viewed the city? To answer these questions, we present some cases of the reception of different works that depicted the city. What interests us in these works is the reactions they inspired among Brazilians and Europeans. These examples 
allow us to observe how their viewpoints were influenced reciprocally. It can be seen how the desire to be like Europe and the valuing of picturesque aspects were instrumental in the formation of a national Brazilian identity.

INDEX

Keywords : Rio de Janeiro, representation, exoticism, civilization, nineteenth century Mots-clés : Rio de Janeiro, représentation, exotisme, civilisation, XIXe siècle

\section{AUTEUR}

\section{ANA MARIA TAVARES CAVALCANTI}

Ana Maria Tavares Cavalcanti, docteur en histoire de l'art (Université Paris 1 PanthéonSorbonne, 1999), est professeur d'histoire de l'art à l'École des Beaux-Arts de l'Universidade Federal do Rio de Janeiro (UFRJ), Brésil. Ses recherches portent sur l'art au Brésil du XIX au début $\mathrm{du} \mathrm{xx}^{\mathrm{e}}$ siècle, la critique d'art, la réception des œuvres d'art, les Salons et les relations artistiques franco-brésiliennes.

ana.canti@eba.ufrj.br 